Ensino

\title{
As Infecções Sexualmente Transmissíveis em livros didáticos de biologia do ensino médio: uma análise de conteúdo
}

\author{
Sexually Transmitted Infections in high school biology textbooks: \\ a content analysis
}

\author{
Halana Rafaela Alves da Silva'(D), Thaís Soares da Silva"i(D, Gemilton de Freitas \\ Mesquita'(i), Isabella Macário Ferro Cavalcanti"'i(i) \\ ' Universidade Federal de Pernambuco, Vitória de Santo Antão, PE, Brasil \\ "Universidade Federal Rural de Pernambuco, Programa de Pós Graduação em Ensino de Ciências e \\ Matemática, Vitória de Santo Antão, PE, Brasil \\ I' Universidade Federal de Pernambuco, Laboratório de Imunopatologia Keizo Asami. Laboratório de \\ Microbiologia e Imunologia, Centro Acadêmico de Vitória, Vitória de Santo Antão, PE, Brasil
}

\section{RESUMO}

O objetivo desse estudo foi analisar o conteúdo ISTs nas coleções didáticas de Biologia de 2018 preconizadas pelo Programa Nacional do Livro Didático do Ensino Médio (PNLEM) do Ministério da Educação. Foram selecionadas e analisadas 8 coleções didáticas de biologia do PNLEM. A metodologia do estudo foi a análise de conteúdo temática, com a definição de cinco categorias a priori: localização do tema; estrutura e formatação; conteúdo; linguagem e recursos visuais que foram divididas em critérios e subcritérios. De acordo com a análise feita, dois livros destacaram-se positivamente, pois, apresentaram melhor desenvolvimento da temática das ISTs. Os demais livros avaliados não abordaram o tema à contento, sendo falhos/superficiais, o que evidencia que o tema ISTs ainda pode ser melhor explorado em alguns livros didáticos de biologia no Ensino Médio. Portanto, ainda assim é importante estimular os professores a explorarem melhor o assunto com seus alunos, indo além dos livros didáticos. Com isso, o estudo concluiu que a maioria dos livros ensino médio preconizados pelo PNLEM não aborda o tema ISTs de forma eficaz, gerando a necessidade de um planejamento didático complementar por parte dos professores no intuito de ampliar o debate desse assunto em sala de aula.

Palavras-chave: Ensino; ISTs; Análise de livro

\section{ABSTRACT}

The aim of this study was to analyze the content of STIs in the didactic collections of Biology of 2018 recommended by the National High School Textbook Program (NHSTP) of the Ministry of Education. Eight didactic collections of biology of NHSTP were selected and analyzed. The methodology of the study was 
thematic content analysis, with the definition of five categories a priori: location of the theme; structure and formatting; content; language and visual resources that were divided into criteria and subcriteria. The other books evaluated did not address the subject to the satisfaction, being flawed/superficial, which shows that the theme STIs can still be better explored in some biology textbooks in high school. Therefore, it is still important to encourage teachers to explore the subject better with their students, going beyond textbooks. Thus, the study concluded that most of the high school books recommended by NHSTP do not address the theme STIs effectively, generating the need for complementary didactic planning by teachers in order to broaden the debate on this subject in the classroom.

Keywords: Teaching; STIs; Book analysis

\section{INTRODUÇÃO}

As Infecções Sexualmente Transmissíveis (ISTs) são causadas por diversos microrganismos transmitidos normalmente por contato sexual sem proteção e de forma ocasional, ou ainda por via sanguínea (BRASIL, 2018). A nomenclatura IST passou a substituir a expressão Doenças Sexualmente Transmissíveis (DSTs), porque existe a possibilidade de uma pessoa ter e transmitir uma infecção, mesmo sem sinais e sintomas (BRASIL, 2015, 2018). Adicionalmente, a transmissão de uma IST também pode acontecer da mãe para a criança durante a gestação, pelo parto ou amamentação (BRASIL, 2014).

As principais ISTs são as infecções causadas pela bactéria Treponema pallidum e pelos vírus da imunodeficiência humana (HIV), papiloma vírus Humano (HPV), vírus de herpes simples (HSV) e vírus da hepatite $B$ e hepatite C (HBV e HCV, respetivamente) (VILLEGAS-CASTANO; TAMAYO-ACEVEDO, 2016). A sífilis é uma IST curável causada pela bactéria Treponema pallidum e pode apresentar várias manifestações clínicas (sífilis primária, secundária, latente e terciária), além de ser transmitida para a criança durante a gestação ou parto (BRASIL, 2018; DA SILVA PEREIRA et al., 2018). O HIV é o vírus causador da Síndrome da Imunodeficiência Adquirida (AIDS) que ataca o sistema imunológico, responsável por defender o organismo de doenças (COMPARINI et al., 2017). A diferença entre "doente de AIDS" e "portador do HIV" está na presença ou não dos sintomas da doença (BRASIL, 2018). O HPV é um vírus que infecta a pele ou mucosas oral, genital ou anal provocando verrugas ano genitais, além de poder provocar o câncer de colo de útero (OPAS/OMS 
(2016). A herpes é causada pelo HSV tipo 1 (HSV1) ou o tipo 2 (HSV2) e possui como principais particularidades as lesões purulentas e dolorosas (ALVES et al., 2017). As Hepatites virais são inflamações no fígado, causadas pelos vírus do gênero Hepatovírus, que nem sempre apresenta sintomas, porém quando os sintomas estão presentes podem ser dor abdominal, fadiga, náusea e amarelamento de pele e olhos (OPAS/OMS, 2016).

Essas infecções por sua vez, estão entre os problemas mais relevantes de saúde pública em todo o mundo, ganhando importância após a epidemia da AIDS na década de 1980 (BRASIL, 2016). No Brasil, ações para a redução da incidência de ISTs permitiram o desenvolvimento de um Programa Nacional de ISTs e AIDS que desenvolve atividades que promovem a saúde e a prevenção das ISTs (BRASIL, 1999).

Devido ao aumento da incidência das ISTs, além das práticas sexuais entre jovens acontecerem cada vez mais cedo, faz-se necessária à articulação da saúde com a educação na tentativa de prevenção dessas ISTs (CASEMIRO et al., 2014). A escola é um espaço primordial para o desenvolvimento do conhecimento, portanto alinhar escola e saúde faz-se cada vez mais necessário para formar um sujeito integral, ou seja, mais crítico e consciente. Assim, a incorporação dessa temática é necessária, pois, o acesso à educação melhora os níveis de saúde. Dessa forma, a contribuição da escola é essencial, pois a mesma assume tanto um papel educativo quanto assistencial (PAES; PAIXÃO, 2016).

A incorporação destas práticas no cotidiano das escolas, por tentativas de aproximações entre as ações dos Ministérios da Saúde e da Educação, torna-se possível a partir do surgimento de vertentes teórico-críticas na análise das proposições de ações educativas no campo da saúde, ou no âmbito da denominada Educação em Saúde (CASEMIRO et al., 2014). A articulação entre os campos da saúde e da educação potencializou o desenvolvimento de ações de promoção da saúde, que refletiram na inclusão de temáticas transversais como saúde e sexualidade no currículo das escolas (MARINHO et al., 2015). A inclusão desses temas se concretizou a partir da apresentação 
dos Parâmetros Curriculares Nacionais (PCNs) como um referencial para o ensino de todo o país (BRASIL, 1997, 1998).

Entretanto, as experiências pedagógicas indicam que o enfoque da sexualidade no âmbito da educação precisa ser prática, para que seja tratada de forma simples e direta, ao mesmo tempo flexível, permitindo que os conteúdos referentes à temática sejam incluídas de forma sistemática beneficiando a aprendizagem e desenvolvimento crescentes (SOUZA, COAN, 2013; NEVES, 2014).Portanto, de acordo com os (PCNs) a educação sexual é um tema que, por fazer parte da existência humana, precisa estar contemplado nos livros didáticos (ALTMANN, 2001), sendo positivo incluir a temática nos currículos escolares a nível nacional (PALMA et al., 2015).

O livro didático faz parte da contemporaneidade da educação brasileira e é o recurso didático mais presente nas salas de aula (ROSA, MOHR, 2010; MUNAKATA, 2016). Esses livros têm um papel muito importante e são bastante valorizados porque dão suporte de apoio no ensino-aprendizagem. Deste modo, para poder exercer seu papel junto à escola, o livro segundo o MEC, precisa estar sempre atualizado e contextualizado (BRASIL, 1994; SCHIMER; SAUERWEIN, 2017).

Historicamente, os livros didáticos têm sido compreendidos como agentes determinantes de currículos, além de possibilitarem a contextualização do conhecimento, porém muitas vezes o conteúdo dos livros se limita a memorização, com raros casos de contextualização, o que distancia os conteúdos da realidade dos alunos e logo formam indivíduos que apenas repetem os conceitos, mas são incapazes de associá-los ao seu cotidiano. Além destas dificuldades muitas vezes os livros empregam conceitos equivocados e até mesmo posições discriminatórias (BRASIL, 2010; LUDOVICO; MAISTRO, 2017).

Nesse sentido, é importante colocar que os PCNs preveem que as questões relacionadas à sexualidade sejam trabalhadas nas escolas, no intuito de auxiliar no conhecimento e também combate à violência e à discriminação. Assim, espera-se que a 
possibilidade da discussão sobre sexualidade possa ampliar o modo de entendimento de crianças e adolescentes sobre o assunto (PALMA et al., 2015).

Desta forma, o objetivo deste estudo é analisar o conteúdo ISTs/DSTs nas coleções didáticas de Biologia do Programa Nacional do Livro Didático do Ensino Médio (PNLEM) do Ministério da Educação, com o intuito de compreender como a temática IST's vem sendo abordada nos livros didáticos, e consequentemente no currículo escolar no Brasil.

\section{MATERIAL E MÉTODOS}

\subsection{Tipo de estudo}

O presente estudo é caracterizado como uma pesquisa qualitativa de acordo com Minayo e colaboradores (2002) baseado na análise do tema ISTs em livros didáticos. Segundo esses autores, a pesquisa qualitativa tem por características a objetivação do fenômeno e a hierarquização das ações de descrever, compreender e explicar um determinado assunto.

Assim, nesse estudo a pesquisa qualitativa se justifica pela intenção de entender o material de forma descritiva, cujo objetivo é explorar como a temática é abordada na coleção de livros didáticos. Ainda segundo esses autores, dentro da pesquisa qualitativa existem diversas subdivisões, tendo sido optado aqui pela análise documental, por se entender que o livro didático é um documento detentor de diversas características, visto serem expressos nele um conjunto de saberes sobre determinado conteúdo.

De acordo com Sá-Silva, Almeida e Guindani (2009), a análise documental tem por objetivo identificar informações factuais nos documentos analisados a partir de questões e hipóteses de interesse. É uma técnica que se utiliza de documentos originais, que ainda não receberam tratamento analítico por nenhum autor. No caso do presente estudo, esses documentos são os livros didáticos. Esses mesmos autores explicam que 
quando um pesquisador utiliza documentos com o intuito de extrair dele informações, ele o faz investigando, examinando, utilizando técnicas apropriadas para seu manuseio e análise. Assim, o pesquisador segue etapas e procedimentos, organiza informações a serem categorizadas e posteriormente analisadas e, por fim, elabora sínteses, ou seja, na realidade, as ações do pesquisador estão impregnadas de aspectos metodológicos, técnicos e analíticos para prosseguir com a análise dos documentos (SÁ-SILVA; ALMEIDA; GUINDANI, 2009).

\subsection{Obtenção dos Livros Didáticos}

Para a construção do corpus empírico foi utilizado como material de análise as 8 coleções didáticas de Biologia do Ensino Médio preconizadas pelo PNLEM do Ministério da Educação (BRASIL, 2017). As editoras consultadas foram a Moderna, Quinteto, SM, FTD, Ática, AJS e Saraiva. Os livros foram identificados utilizando letras de A a H (Quadro 1).

QUADRO 1 - Identificação dos livros de Biologia do PNLEM em 2018

\begin{tabular}{|c|c|c|c|}
\hline Livro & Autor & Editora & Ano \\
\hline $\begin{array}{l}\text { A) Bio, } 3^{\circ} \text { Ano do ensino médio } \\
\text { (volume } 3 \text { ) }\end{array}$ & Sônia Lopes, Sergio Rosso & Saraiva & 2016 \\
\hline $\begin{array}{l}\text { B) Biologia vol. } 3,3^{\circ} \text { Ano do ensino } \\
\text { médio (volume } 3 \text { ) }\end{array}$ & Vivian L. Mendonça & AJS & 2016 \\
\hline $\begin{array}{l}\text { C) Biologia hoje, } 1^{\circ} \text { Ano do ensino } \\
\text { médio (volume } 1 \text { ) }\end{array}$ & $\begin{array}{l}\text { Sérgio Linhares, Fernando Gewandsznajder, } \\
\text { Helena Pacca }\end{array}$ & Ática & 2016 \\
\hline $\begin{array}{l}\text { D) Conexões com a biologia, } 1^{\circ} \text { Ano } \\
\text { do ensino médio (volume } 1 \text { ) }\end{array}$ & Miguel Thompson, Eloci Peres Rios & Moderna & 2016 \\
\hline $\begin{array}{l}\text { E) Biologia unidade e diversidade, } 2^{\circ} \\
\text { Ano do ensino médio (volume } 2 \text { ) }\end{array}$ & José Arnaldo Favaretto & FTD & 2016 \\
\hline $\begin{array}{l}\text { F) Ser Protagonista, } 1^{\circ} \text { Ano do } \\
\text { ensino médio (volume } 1 \text { ) }\end{array}$ & $\begin{array}{l}\text { André Catani, Elisa Garcia Carvalho, } \\
\text { Fernando Santiago dos Santos, João Batista } \\
\text { Aguilar, Silvia Helena de Arruda Campos }\end{array}$ & SM & 2016 \\
\hline $\begin{array}{l}\text { G) \# Contato Biologia, } 1^{\circ} \text { Ano do } \\
\text { ensino médio (volume } 1 \text { ) }\end{array}$ & Marcela Ogo, Leandro Godoy & Quinteto & 2016 \\
\hline $\begin{array}{l}\text { H) Biologia Moderna, } 1^{\circ} \text { Ano do } \\
\text { ensino médio (volume } 1 \text { ) }\end{array}$ & $\begin{array}{l}\text { José Mariano Amabis, Gilberto Rodrigues } \\
\text { Martho }\end{array}$ & Moderna & 2016 \\
\hline
\end{tabular}

Fonte: Autor, 2019 


\subsection{Definição dos instrumentos e procedimentos para análise do corpus empírico}

O presente estudo foi desenvolvido através da análise de conteúdo baseado em Bardin (2016), que a define como um conjunto de instrumentos metodológicos, que se aplica a discursos ou conteúdos extremamente diversificados. O fator comum entre essas técnicas é uma hermenêutica controlada, baseada na dedução ou inferência. Trata-se de um esforço de interpretação que oscila entre dois polos, indo do rigor da objetividade até a fecundidade da subjetividade (BARDIN, 2016).

Moraes (1999) explica a análise de conteúdo como uma metodologia de pesquisa usada para descrever e interpretar o conteúdo de toda classe de documentos e textos. Trata-se de uma análise que conduz descrições sistemáticas, qualitativas ou quantitativas, com o objetivo de ajudar a reinterpretar as mensagens presentes no que se analisa, e a atingir uma compreensão maior de seus significados em um nível que vai além de uma leitura comum. Existem vários métodos para a realização de uma análise de conteúdo, e esse estudo utilizou a análise de conteúdo temática, que consiste em categorizar e organizar os enunciados de acordo com as categorias. A análise de conteúdo temática é dividida em 3 etapas: leitura flutuante, categorização (que pode ser a priori ou a posteriori) e análise e interpretação.

Nesse estudo, as categorias foram definidas a priori, dessa forma, buscou-se, através da análise de critérios pré-definidos, criar um sistema de categorias que representem da melhor maneira possível os elementos que serão analisados, cujo objetivo principal é investigar como o conteúdo IST é abordado nos livros didáticos em questão.

Nesse estudo, os critérios para a análise foram elaborados com base em pesquisas semelhantes às encontradas nos trabalhos de Vasconcelos e Souto (2003), Espínola (2007), Rosa e Mohr (2010) e Batista, Cunha e Cândido (2010). Também é importante ressaltar que os Parâmetros Curriculares Nacionais do Ensino Médio PCNEM (BRASIL, 2002) ajudaram a elencar esses critérios, já que esse documento é um norteador dos conteúdos que devem ser abordados em cada disciplina. 
A partir desta análise prévia dos livros foram selecionadas cinco categorias: 1 . Localização; 2. Estrutura e formatação; 3. Conteúdo; 4. Linguagem e 5. Recursos visuais. Essas categorias foram divididas em critérios e subcritérios para realizar uma maior exploração do conteúdo dos livros (Quadro 2). Após a definição dos critérios foi realizada uma leitura para identificar as categorias citadas no quadro 2. Posteriormente as informações foram catalogas em planilhas para identificar como a temática IST era abordada nos livros analisados.

QUADRO 2 - Categorias e tópicos de análise.

\begin{tabular}{|c|c|}
\hline Categorias & Tópico de análise \\
\hline Localização & Seres vivos; Fisiologia reprodutiva. \\
\hline $\begin{array}{l}\text { Estrutura e } \\
\text { Formatação }\end{array}$ & $\begin{array}{l}\text { Presença de textos complementares; Cita autores e colaboradores; Cita outras fontes } \\
\text { de informação; Ilustração com referência a programas oficiais de saúde; Número de } \\
\text { páginas; Quantidade de ISTs abordadas nos capítulos. }\end{array}$ \\
\hline Conteúdo & $\begin{array}{l}\text { Presença de conceitos básicos ou pré-requisitos para compreensão; Aborda as } \\
\text { Infecçôes Sexualmente Transmissíveis mais frequentes no país; O capítulo aborda } \\
\text { prevenção e tratamento das ISTs; Informações complementares atrativas para alunos } \\
\text { de ensino médio; Contextualização biológica ou ciências sociais. }\end{array}$ \\
\hline Linguagem & Presença de clareza e objetividade; Presença de estereótipos e situações caricatas. \\
\hline $\begin{array}{l}\text { Recursos } \\
\text { visuais }\end{array}$ & $\begin{array}{l}\text { Ausência ou presença de ilustrações; Tipos de ilustrações; Layout atraente, organizado } \\
\text { e pertinente; Indicação da referência da ilustração; Coerência com conteúdo; } \\
\text { Propagandas do MS. }\end{array}$ \\
\hline
\end{tabular}

Fonte: Autor, 2019

\section{RESULTADOS E DISCUSSÃO}

Apesar de todos os recursos didáticos inovadores proporcionados pelas novas tecnologias, o livro ainda é o principal recurso utilizado nas escolas (MARTINS, SANTOS, EL-HANI, 2012; ALVES; RODRIGUES; SANTOS, 2018). Portanto, diante da necessidade de compreensão da relação entre saúde e questões biológicas, sociais e ambientais, se torna fundamental averiguar o tratamento dos conteúdos relativos à saúde pelos livros didáticos. Nesse contexto o presente trabalho teve como objetivo analisar como o livro didático contribui para a sensibilização e conscientização crítica do estudante em relação a temática IST e aos fatores que intervêm positiva e negativamente a sua saúde. 
Nessa perspectiva os livros didáticos foram analisados sobre a ótica de quatro categorias: localização, estrutura e formatação conteúdo, linguem e recursos visuais. Os resultados da análise do presente estudo sobre as categorias avaliadas nos livros de biologia do ensino médio estão descritos no Quadro 3, com exceção do livro A (AMABIS; MARTHO, 2016), visto que o mesmo não aborda a temática ISTs/DSTs.

Em relação ao livro A (AMABIS; MARTHO, 2016), que não aborda a temática ISTs/DSTs, em seu volume 1, o capítulo 11 é destinado à reprodução humana, um capítulo interessante para abordar a temática. Nesse capítulo são abordados os seguintes tópicos: bebês de proveta e clonagem humana; sistema genital feminino; sistema genital masculino; hormônios relacionados à reprodução; gravidez e parto. Em nenhum deles o tema ISTs foi mencionado. O capítulo também traz, como textos auxiliares: Homens e mulheres: compreender conceitos para vencer preconceitos e estereótipos; Compartilhando o útero materno: gêmeos humanos; Controle da reprodução humana. Em meio a todo esse conteúdo destinado à reprodução humana, não há nenhum tópico destinado às ISTs.

O volume 2 do citado livro traz, entre outros conteúdos, informações sobre vírus (capítulo 2), bactérias (capítulo 2), protozoários (capítulo 3) e fungos (capítulo 3), onde seria importante e pertinente aproveitar para abordar a temática das ISTs, porém praticamente não há citações sobre essas infecções. No âmbito das doenças virais, os autores dão ênfase para a gripe e a AIDS, através de textos complementares. Sobre doenças bacterianas, estas são citadas de forma muito sutil em um texto complementar que explana sobre a importância das bactérias para a humanidade, todavia não cita nada sobre as bactérias causadoras de ISTs. O conteúdo sobre doenças por protozoários é mais amplo, sendo desenvolvido em 6 páginas, abordando os protistas em texto auxiliar, bem ilustrado e com esquemas, fotos, desenhos e infográficos, contudo não envolve nenhuma IST. Nesse livro são trabalhadas apenas a amebíase, leishmaniose, doença de Chagas e malária, trazendo ao final uma atividade com 17 questões voltadas para as doenças causadas por estes protozoários. Sobre as doenças causadas por fungos, há ausência de informações. Desta forma, há uma carência de informações sobre ISTs tanto no volume 1 como no volume 2 dessa coleção de livros didáticos. 


\section{QUADRO 3 - Análise dos livros de biologia do ensino médio sobre o tema Infecções}

\section{Sexualmente Transmissíveis}

\begin{tabular}{|c|c|c|c|c|c|}
\hline Categorias & Critérios de análise & Livro B & Livro C & Livro D & Livro E \\
\hline \multirow{5}{*}{ Localização } & Seres vivos & Ausente & Presente & Ausente & Ausente \\
\hline & Fisiologia reprodutiva & Presente & Presente & Presente & Presente \\
\hline & $\begin{array}{l}\text { Presença de textos } \\
\text { complementares }\end{array}$ & 0 & 0 & 1 & 0 \\
\hline & $\begin{array}{l}\text { Cita autores e } \\
\text { colaboradores }\end{array}$ & 0 & 0 & 0 & 0 \\
\hline & $\begin{array}{l}\text { Cita outras fontes de } \\
\text { informação } \\
\text { llustração com referência }\end{array}$ & Não & Sim & Sim & Não \\
\hline \multirow[t]{5}{*}{$\begin{array}{l}\text { Estrutura e } \\
\text { formatação }\end{array}$} & $\begin{array}{l}\text { a programas oficiais de } \\
\text { saúde }\end{array}$ & Não & Sim: AIDS, HPV & Sim: HPV & Não \\
\hline & $\mathrm{N}^{\circ}$ de páginas & 1 & Vol. $2=5 /$ Vol. $.3=2$ & 2 & 5 \\
\hline & $\begin{array}{l}\text { Quantidade de ISTs } \\
\text { abordadas nos } \\
\text { capítulos. }\end{array}$ & $\begin{array}{l}9 \text { Sífilis, gonorreia, } \\
\text { cancro mole, HPV, LGV, } \\
\text { pediculose pubiana, } \\
\text { AIDS, tricomoníase e } \\
\text { herpes genital }\end{array}$ & $\begin{array}{l}9 \text { HPV, gonorreia, } \\
\text { hepatite C, herpes } \\
\text { genital, LGV, síflis, AIDS, } \\
\text { tricomoníase e } \\
\text { candidíase }\end{array}$ & $\begin{array}{l}7 \text { Gonorreia, clamídia, } \\
\text { HPV, síflis, candidíase, } \\
\text { herpes genital e AIDS }\end{array}$ & $\begin{array}{l}10 \mathrm{HPV} \text {, gonorreia, } \\
\text { AIDS, clamídia, } \\
\text { candidíase, pediculose } \\
\text { pubiana, śfilis, herpes } \\
\text { genital, hepatite Be } \\
\text { tricomoníase }\end{array}$ \\
\hline & $\begin{array}{l}\text { Presença de conceitos } \\
\text { básicos ou pré-requisitos } \\
\text { para compreensão }\end{array}$ & Sim & Sim & Sim & Sim \\
\hline & $\begin{array}{l}\text { Aborda as Infecções } \\
\text { Sexualmente } \\
\text { Transmissíveis mais } \\
\text { frequentes no país }\end{array}$ & $\begin{array}{l}\text { Sim: síflis, gonorreia, } \\
\text { cancro mole, } \\
\text { linfogranulo venéreo, } \\
\text { condiloma acuminado, } \\
\text { pediculose pubiana, } \\
\text { tricomoníase, AIDSe } \\
\text { herpes genital }\end{array}$ & $\begin{array}{l}\text { Sim: condiloma } \\
\text { acuminado, gonorreia, } \\
\text { hepatite C, herpes } \\
\text { genital, linfogranuloma } \\
\text { venéreo, síflis, AIDS, } \\
\text { tricomoníase e } \\
\text { candidíase }\end{array}$ & $\begin{array}{l}\text { Sim: gonorreia, } \\
\text { clamídia, condiloma } \\
\text { acuminado, sífilis, } \\
\text { candidíase, herpes } \\
\text { genital e AIDS }\end{array}$ & $\begin{array}{l}\text { Sim: gonorreia, } \\
\text { clamídia, candidíase, } \\
\text { síflis, herpes genital, } \\
\text { condiloma acuminado, } \\
\text { hepatite B, } \\
\text { tricomoníase, } \\
\text { pediculose pubiana e } \\
\text { AIDS }\end{array}$ \\
\hline \multirow[t]{4}{*}{ Conteúdo } & $\begin{array}{l}\text { O capítulo aborda } \\
\text { prevenção e tratamento } \\
\text { das ISTs }\end{array}$ & Não & $\begin{array}{l}\text { Sim: condiloma } \\
\text { acuminado, gonorreia, } \\
\text { hepatite C, herpes } \\
\text { genital, LGV, síflis, AIDS, } \\
\text { tricomoníase, } \\
\text { candidíase }\end{array}$ & $\begin{array}{l}\text { Sim: gonorreia, } \\
\text { clamídia, condiloma } \\
\text { acuminado, sífilis, } \\
\text { candidíase, herpes } \\
\text { genital e AIDS }\end{array}$ & $\begin{array}{l}\text { Sim: gonorreia, clamídia, } \\
\text { candidíase, síflis, herpes } \\
\text { genital, condiloma } \\
\text { acuminado, hepatite B, } \\
\text { tricomoníase, } \\
\text { pediculose pubiana e } \\
\text { AIDS }\end{array}$ \\
\hline & $\begin{array}{l}\text { Informações } \\
\text { complementares atrativas } \\
\text { para alunos de ensino } \\
\text { médio }\end{array}$ & Não & $\begin{array}{l}\text { Sim: Indicação de livros } \\
\text { e sites com maiores } \\
\text { informações }\end{array}$ & Não & Não \\
\hline & $\begin{array}{l}\text { Contextualização biológica } \\
\text { ou ciências sociais }\end{array}$ & Não & $\begin{array}{l}\text { Sim: Biológica - ciclo da } \\
\text { doença }\end{array}$ & Não & $\begin{array}{l}\text { Sim: Biológica - ciclo da } \\
\text { doença }\end{array}$ \\
\hline & $\begin{array}{l}\text { Presença de clareza e } \\
\text { objetividade }\end{array}$ & Sim & Sim & Sim & Sim \\
\hline \multirow[t]{2}{*}{ Linguagem } & $\begin{array}{l}\text { Presença de estereótipos } \\
\text { e situações caricatas }\end{array}$ & Não & Não & Não & Não \\
\hline & $\begin{array}{l}\text { Ausência ou presença de } \\
\text { ilustrações }\end{array}$ & Ausente & Presente & Presente & Presente \\
\hline \multirow[t]{2}{*}{$\begin{array}{l}\text { Recursos } \\
\text { visuais }\end{array}$} & Tipos de ilustrações & Ausente & $\begin{array}{l}\text { Esquemas dos } \\
\text { patógenos, ciclo } \\
\text { reprodutivo da AIDS e } \\
\text { campanhas do MS }\end{array}$ & $\begin{array}{l}\text { Propaganda do MS } \\
\text { sobre HPV e o laço } \\
\text { vermelho da } \\
\text { campanha da AIDS }\end{array}$ & $\begin{array}{l}\text { Esquemas dos } \\
\text { patógenos e ciclo } \\
\text { reprodutivo da AIDS }\end{array}$ \\
\hline & $\begin{array}{l}\text { Layout atraente, } \\
\text { organizado e pertinente }\end{array}$ & Não & Sim & Não & Sim \\
\hline
\end{tabular}

Continua... 


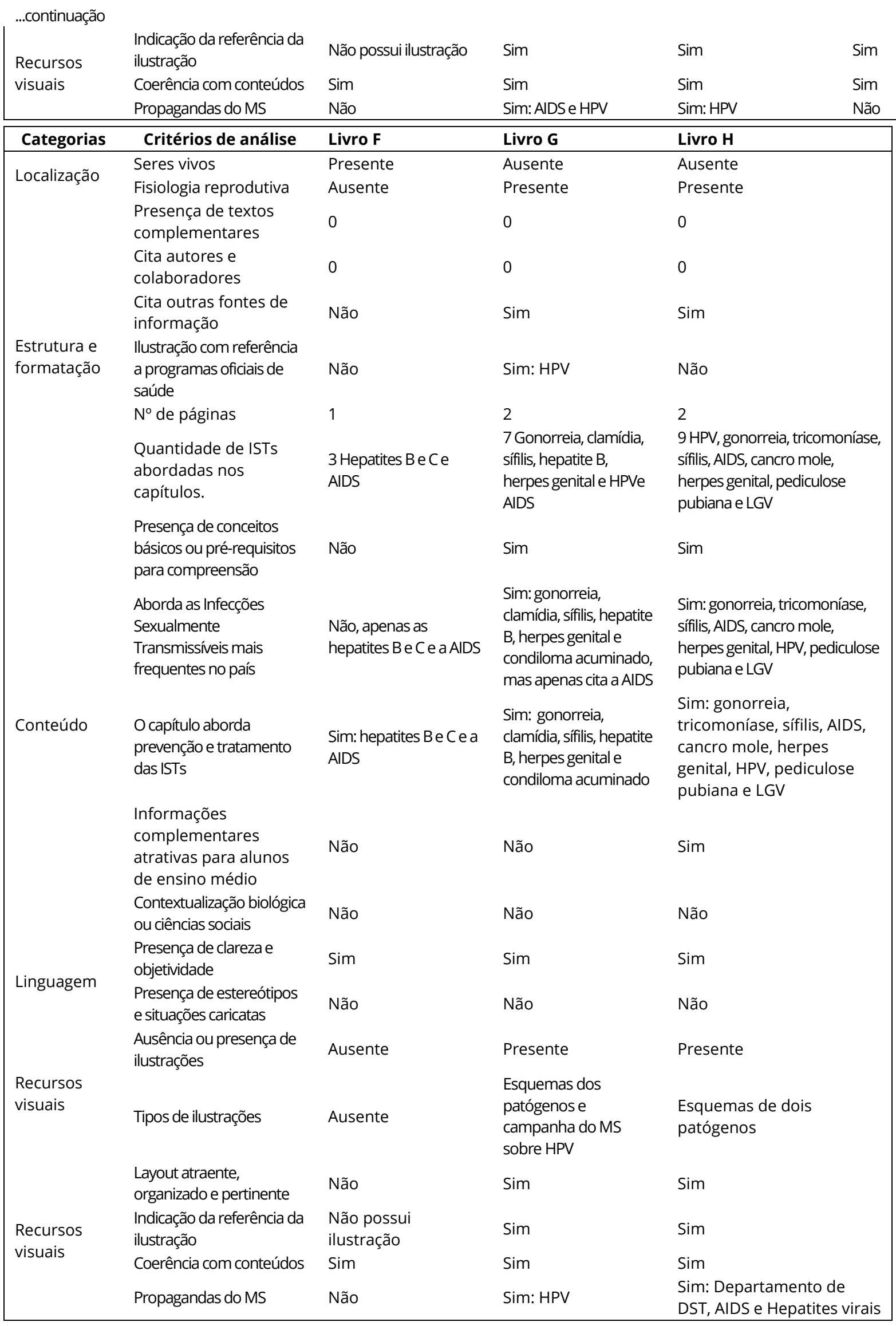

Fonte: Autor, 2019

AIDS: Síndrome da Imunodeficiência Adquirida; HPV: Papiloma Vírus Humano; ISTs: Infecções Sexualmente Transmissíveis; LGV: Linfogranuloma Venéreo; Vol.: volume 
Em relação ao livro B, (LOPES, ROSSO, 2016) o volume 3, unidade 1 - Espécie Humana, em seu capítulo 1 sobre reprodução e desenvolvimento embrionário humano, traz o item 6 dedicado exclusivamente às ISTs (LOPES, ROSSO, 2016). Esse item, apesar de curto e sucinto, explica o que são as DSTs (não utiliza a nomenclatura ISTs) e alerta sobre seus riscos. O item traz ainda explicações básicas sobre sífilis, gonorreia, cancro mole, linfogranuloma venéreo (clamídia), condiloma acuminado (HPV), pediculose pubiana e tricomoníase, em apenas uma página, e cita a AIDS e o herpes genital. Ao final do capítulo, traz questões de múltipla escolha sobre os temas abordados e uma delas é sobre as DSTs. Entretanto, não aborda questões relacionadas com a prevenção das ISTs, não traz recursos visuais ou textos complementares, nem mesmo indicação de outras fontes de informação.

O mesmo capítulo 1 traz informações sobre noções gerais da reprodução, gametogênese, sistema genital masculino, sistema genital feminino, fecundação e desenvolvimento embrionário humano. Adicionalmente, um dos temas propostos para discussão é a gravidez na adolescência.

$\mathrm{Na}$ interpretação desse estudo, esse livro, apesar de abordar o tema, o traz de forma muito superficial, deixando de informar sobre prevenção e tratamento de algumas doenças, como a AIDS, a hepatite C e o herpes genital. Portanto, o tema poderia ser melhor aproveitado nesse livro. Para o professor que utiliza esse livro como referência didática em suas aulas, fica a responsabilidade de aprofundar o tema com seus alunos em classe.

O livro C (MENDONÇA, 2016) já se mostra um pouco mais completo, trazendo em seu volume 3, capítulo 5, sobre controle hormonal e reprodução, o item 4 sobre reprodução humana, que aborda o sistema genital feminino, sistema genital masculino, ato sexual, DSTs e métodos anticoncepcionais. O tópico sobre DSTs possui duas páginas, e explica o que são as DSTs, seus riscos, tratamentos e como preveni-las, além de trazer uma tabela explicativa com dados sobre etiologia, sintomas e tratamento sobre condiloma acuminado, gonorreia, hepatite C, herpes genital, linfogranuloma venéreo, sífilis, AIDS e tricomoníase. Também há um texto sobre a candidíase. Logo abaixo da 
tabela segue uma informação de que o volume 2 do livro traz dados que aprofundam as informações sobre estas DSTs. Ao final do capítulo traz exercícios, porém nenhum deles aborda as ISTs/DSTs.

Em relação ao volume 2 do livro de Linhares e colaboradores. (2016), as ISTs são abordadas nos capítulos referentes aos vírus (capítulo 2 - AIDS e HPV), moneras bactérias (capítulo 3 - sífilis), protistas (capítulo 4 - tricomoníase). Nesses capítulos essas doenças são bem mais detalhadas, havendo aproveitamento de recursos visuais e indicações de outras fontes de informação para aprofundar o conhecimento, inclusive sites oficiais do governo. O livro, em ambos os volumes avaliados, é muito ilustrado, com linguagem clara e objetiva.

O livro D aborda o tema em sua unidade 6 - Saúde: bem-estar físico, mental e social, tema 3, que fala sobre os principais tipos de doenças, trazendo 6 classificações, sendo a última as DSTs (THOMPSON, RIOS, 2016). Neste livro são dedicadas duas páginas ao tema, que traz informações sobre o que são as DSTs e como preveni-las, abordando em especial gonorreia, clamídia, condiloma acuminado, sífilis, candidíase, herpes genital e AIDS, além de trazer a propaganda do MS sobre a vacinação contra o HPV. O livro sugere atividades a serem feitas em sala de aula, sendo uma delas em relação às DSTs. Porém, as informações trazidas são muito superficiais e sem textos complementares nem indicação de leituras adicionais. Entretanto, o livro é ricamente ilustrado, apesar de no tema deste estudo não trazer ilustrações, apenas a propaganda do governo sobre o HPV. Inclusive, no tema seguinte, sobre tecnologias na saúde, traz dados sobre métodos contraceptivos, com ilustrações informativas.

O livro E (LINHARES et al., 2016) em compensação, mostra-se bem mais completo, dedicando 5 páginas ao assunto, que é abordado no capítulo 12 , sobre reprodução. 0 capítulo traz também os tópicos reprodução humana e métodos anticoncepcionais. No tópico sobre as DSTs, os autores explicam o que são essas doenças, como prevenir, como identificar sinais e sintomas e a importância em buscar opinião médica para o diagnóstico. O livro traz ainda ilustrações e informações sobre gonorreia, clamídia, candidíase, sífilis, herpes genital, condiloma acuminado, hepatite B, tricomoníase, 
pediculose pubiana e AIDS. Os autores abordam de forma clara e objetiva estas doenças, dedicando especial atenção à AIDS.

No final do capítulo tem algumas questões e sugestões de atividades que incluem o tema das DSTs, como a sugestão de dividir a turma em grupos e indicar a pesquisa dos temas do capítulo em livros e internet, ou mesmo a partir de entrevistas com médicos e outros profissionais de saúde. O livro também sugere os temas: Quais são as principais formas de transmissão da AIDS no Brasil e como ela está evoluindo no país? Quais são as principais doenças sexualmente transmissíveis no Brasil e quais os fatores que provocam o aumento da ocorrência dessas doenças? Quais os exames e cuidados recomendados para os jovens se prevenirem contra as DSTs?

Esse livro abordou com rico conteúdo o tema das ISTs, explorando o assunto de forma integral, tendo a preocupação de enriquecer o estudo com recursos visuais e propondo exercícios. O único ponto negativo foi a ausência da indicação de fontes alternativas de pesquisa e de textos complementares.

O livro F (FAVARETTO, 2016) não apresenta quase nenhum conteúdo sobre ISTs/DSTs. Esse livro traz apenas uma tabela sobre "Doenças humanas causadas por vírus, principais manifestações e formas de prevenção", no capítulo sobre vírus, que contém 13 doenças, entre elas 3 ISTs, as hepatites B e C e a AIDS, com seus respectivos agentes etiológicos, transmissão, manifestações e prevenção. Nos demais capítulos relacionados a microrganismos não há nenhuma referência a ISTs/DSTs.

O livro G aborda o tema em uma sessão dentro do capítulo 4, sobre reprodução e embriologia, logo após o tema de métodos contraceptivos (OGO, GODOY, 2016). Com o título de DSTs, o livro traz informações básicas sobre gonorreia, clamídia, sífilis, hepatite $B$, herpes genital e condiloma acuminado. Esse livro inclui ilustrações sobre os microrganismos, além de uma publicação do MS sobre o HPV. Não traz textos complementares nem indicação de outras fontes de pesquisa sobre o tema. Ao final do capítulo traz um exercício sobre DSTs. O livro é bem objetivo, com linguagem clara sobre o tema, porém ISTs/DSTs é pouco explorado, sendo contemplado em apenas duas páginas. 
O livro H (AMABIS, MARTHO, 2016) avaliado por esse estudo desenvolve o tema em sua unidade 3, capítulo 12, sobre reprodução dos seres vivos, que também explora os tópicos: reprodução; reprodução humana; e métodos contraceptivos. O livro dedica duas páginas ao tema das DST se traz informações sobre gonorreia, tricomoníase, sífilis, AIDS, inclusive com um quadro informando "assim pega" e "assim não pega", cancro mole, herpes genital, HPV, pediculose pubiana, linfogranuloma venéreo. O livro propõe uma atividade de estudo relacionada com as DSTs e, ao final do capítulo, traz um exercício sobre DSTs, além de indicações de leituras e pesquisas para aprofundar os temas do capítulo, recomendando a página do MS para pesquisa sobre DSTs. Apesar de trazer uma linguagem clara e objetiva sobre o tema, e abordar variadas ISTs, o livro H poderia ter aprofundado mais o assunto, ampliando os recursos visuais e trazendo textos complementares.

Em proposta semelhante a desse estudo, os autores De Cicco e Vargas (2012) objetivaram compreender como o tema DSTs é abordado nos livros didáticos de biologia do ensino médio. Os resultados desses pesquisadores apontaram predominância do tema em tópicos de leituras complementares e ênfase na abordagem da AIDS, não encontrando relação explícita entre os conteúdos da biologia e o contexto social. No presente estudo já foram encontroados livros com abordagens específicas direcionadas ao tema. Ao relacionarmos com os resultados obtidos identificamos que, nos livros C e E havia uma atenção especial ao assunto, onde o livro C (MENDONÇA, 2016) aborda o tema tanto no contexto de seres vivos, focando nos patógenos e suas características, como no de fisiologia e reprodução, abordando a questão da transmissão e prevenção, e o livro E (LINHARES et al., 2016) que aprofundou o tema em maiores detalhes, dedicando 5 páginas sobre o assunto, mostrando uma melhora em relação à avaliação dos livros de ensino de biologia realizado em 2012 pelos autores citados, De Cicco e Vargas (2012).

De Cicco e Vargas (2012) alegam ainda que os conteúdos de ISTs estão associados à duas temáticas: seres vivos e fisiologia reprodutiva. Dessa forma, quando o professor trabalha o tema associado aos seres vivos, pode aproveitar para discutir aspectos 
biológicos dos microrganismos e suas implicações na saúde do indivíduo. Da mesma forma, quando o tema é abordado em associação com a fisiologia reprodutiva, o professor pode explorar outras perspectivas associadas à sexualidade dos indivíduos e à prevenção. Nesse sentido, o livro C (MENDONÇA, 2016) foi o que melhor abordou a temática, pois a explorou em ambos os capítulos. Caso não haja a abordagem nas duas frentes, Cicco e Vargas (2012) acreditam ser mais interessante que o conteúdo esteja presente em fisiologia reprodutiva, já que os microrganismos irão se reproduzir e sobreviver no corpo do hospedeiro. Os autores enfatizam ainda que a entrada do patógeno no organismo humano acontece pela via reprodutiva, através do ato sexual, portanto se torna mais importante abordar o tema IST em fisiologia reprodutiva possibilitando abordar o tema em dois momentos, formas de contágio e prevenção das ISTs, além de aproveitar para aprofundar o assunto explicando a patogênese dessas infecções (CICCO, VARGAS, 2012).

Em estudo anterior, Monteiro, Bizzo e Gouw (2010) investigaram quais perspectivas estão mais presentes nos livros didáticos aprovados pelo Programa Nacional do Livro Didático em 2008 para as séries finais do Ensino Fundamental, em relação aos temas relacionados às DSTs e à infecção pelo HIV/AIDS. Segundo os autores, os resultados demonstraram que havia uma predominância de conteúdos relacionados à descrição da doença e do ciclo do agente, configurando o predomínio da perspectiva biomédica. Eles evidenciaram ainda a presença da preocupação com o desenvolvimento de atitudes de prevenção e com a discussão de aspectos culturais relacionados a esses agravos. Esse enfoque traz uma perspectiva interessante que o professor pode abordar em sala de aula, fazendo a ligação das ISTs com os aspectos culturais, procurando posicionar os alunos em relação a essas infecções, dentro de seu contexto social.

O ambiente escolar assim como o livro didático devem incentivar e proporcionar a participação dos alunos, como sujeitos atuantes na construção do conhecimento, para que dessa forma, os alunos sejam motivados a interpretar as relações do homem com o espaço. Nessa perspectiva, se faz necessária a tomada de mudanças no enfrentamento pedagógico, curricular, administrativo e estrutural, nos quais a escola 
está assentada. Torna-se necessário repensar as práticas que estão sendo conduzidas neste ambiente, para fazer sentido o que está sendo posto, aliando as informações disponíveis para construção do conhecimento (ALMEIDA, 2006).

Afinal, conforme defendem Alves, Rodrigues e Santos (2018), o ensino de ciências no ambiente escolar possui o papel de despertar nos estudantes a capacidade de desenvolverem atitudes e habilidades que são imprescindíveis para seu convívio em sociedade, motivo pelo qual a Educação em Saúde tem se tornado uma importante aliada, por favorecer mudanças comportamentais, através da aquisição de novos conhecimentos e adoção de estilos de vida saudáveis com intuito de promoção da saúde individual e coletiva pelos estudantes.

Dessa forma, a investigação de como as temáticas que se relacionam com a saúde vêm sendo desenvolvidas nos livros didáticos se faz necessária, tendo em vista a importância desse recurso para motivar o aprendizado e ajudar os estudantes a se tornarem capazes de adotar práticas comportamentais com base no conhecimento adquirido em sala de aula. O estudo de Alves, Rodrigues e Santos (2018), em abordagem semelhante à da presente pesquisa, realizou uma análise de conteúdo sobre o tema "promoção da saúde" nos livros didáticos de Ciências dos anos finais do Ensino Fundamental, e constatou que, de forma geral, todos os livros avaliados mantiveram o enfoque do tema centrado apenas em aspectos biológicos e fisiológicos no modelo biomédico da saúde, sem considerar os aspectos biopsicossociais dos alunos. Esse enfoque acaba por não contribuir significativamente para uma melhoria e manutenção do bem-estar e da qualidade de vida dos estudantes, visto que não estimulam em sua plenitude, mudanças comportamentais e estilos de vida saudáveis por meio de escolhas conscientes e responsáveis voltadas à saúde.

Essa realidade também foi averiguada na presente análise, verificando-se a necessidade de uma abordagem mais holística, que considere os aspectos biopsicossociais dos alunos com relação aos condicionantes e determinantes da saúde, e não siga mantendo-se fundamentada no modelo sanitarista de saúde, ainda mais em um tema tão importante como é o das ISTs. 
O livro didático é, para alguns professores, um dos poucos materiais didáticos disponíveis, e pode ser para outros a única fonte de informação para alunos e professores. Mas, mesmo sendo um produto desenvolvido para facilitar a compreensão dos alunos, os livros podem oferecer problemas em relação a como apresentar informações novas e de difícil assimilação para os estudantes. Todavia, muitos professores usam o livro didático de forma acrítica, ou seja, sem analisar os conteúdos, as formas de apresentação dos assuntos e suas abordagens metodológicas, sendo, portanto necessária uma constante análise crítica por parte dos educadores sobre o seu conteúdo e a sua pertinência, antes dos mesmos serem utilizados em sala de aula (SANTOS; TERÁN; SILVA-FORSBERG, 2011).

Além disso, é relevante analisar em que medida os livros didáticos utilizados nas escolas proporcionam meios para estimular nos alunos escolhas de hábitos e atitudes saudáveis que, depois, possam repercutir de forma positiva na comunidade, através da referência das próprias transformações provenientes do crescimento e desenvolvimento humanos desses alunos. Nesse caso, torna-se desejável que os livros veiculem conhecimentos que contribuam para o desenvolvimento de ações positivas na comunidade, por ser este o espaço mais efetivo e apropriado para se promover a saúde, ou seja, um dos principais contextos no qual podem ter lugar mudanças sociais com o potencial de melhorar a qualidade de vida dos indivíduos e, consequentemente, da própria comunidade. Portanto, os livros didáticos devem colaborar para uma educação em saúde que crie condições para o desenvolvimento dos estudantes como membros ativos e críticos da sociedade (MARTINS; SANTOS; EL-HANI, 2012).

Por ser o recurso mais utilizado no ensino de ciências, essa centralidade confere ao livro didático estatuto e funções privilegiadas, na medida em que é através dele que o professor organiza, desenvolve e avalia seu trabalho pedagógico de sala de aula. Afinal, as principais lembranças que grande parte das pessoas têm de disciplinas cursadas na escola está relacionada aos livros didáticos (CARNEIRO; SANTOS; MÓL, 2005).

De acordo com os pesquisadores Martins, Santos e El-Hani (2012), e corroborando o presente estudo, existem deficiências em relação a como os conteúdos 
de saúde vêm sendo tratados nos livros didáticos brasileiros, e essas têm sido apontadas por pesquisadores já há muitos anos, especialmente sobre sua desvinculação da realidade e das reais necessidades dos alunos, bem como do uso de metodologias que priorizam a exposição teórica e a prescrição de regras que devem ser seguidas pelos alunos. Com isso, esse material didático termina por dificultar o ensino e a aprendizagem dos conceitos, processos e comportamentos que devem estar envolvidos na manutenção e recuperação da saúde individual e/ou coletiva.

Saúde e biologia estão interligadas, e o ensino da biologia é fundamental para a compreensão dos organismos vivos, de seus funcionamentos e inter-relações, das doenças que se desenvolvem por meio dessas inter-relações, bem como sobre os aspectos relacionados com a manutenção da saúde, de hábitos saudáveis e como se prevenir de certas doenças. Nesse sentido, é importante frisar que a temática IST é uma subtemática de Microbiologia, e que, uma vez que a Microbiologia é pouco abordada em livros didáticos, altera implicitamente a abordagem da temática IST.

\section{CONCLUSÕES}

Essa pesquisa concluiu que entre as opções de livros didáticos avaliadas, os livros C (Biologia, vol. 2 e 3, $3^{\circ}$ Ano do ensino médio de Vivian L. Mendonça) e E (Biologia hoje, $1^{\circ}$ Ano do ensino médio de Sérgio Linhares, Fernando Gewandsznajder, Helena Pacca) parecem apresentar melhor desenvolvimento da temática das ISTs, com destaque para o livro $C$, que explorou o assunto tanto em Seres Vivos como em Fisiologia Reprodutiva.

Ainda assim, é preciso estimular os professores a explorarem melhor o assunto com seus alunos, indo além dos livros didáticos, visto que a maioria deles não aborda o tema à contento, sendo falhos e superficiais. Assim, faz-se necessário um planejamento didático que envolva estratégias complementares na busca de esclarecer aos alunos os riscos envolvidos com as ISTs e sobre a importância da prevenção e da responsabilidade consigo mesmo e com o outro em uma relação à dois. 


\section{REFERÊNCIAS}

ALMEIDA MEB. Tecnologias na educação, formação de educadores e recursividade entre teoria e prática: trajetória do programa de pós-graduação em educação e currículo. Revista ECurriculum. 2006; 1(1):1-28.

ALTMANN, H. Orientação sexual nos Parâmetros Curriculares Nacionais. Revista Estudos Feministas. 2009;9 (2): 575-585.

ALVES HHS, SILVA BN, PEREIRA SES, FERREIRA SCH, JUSTINO ICB. Clínica e diagnóstico das infecções pelos vírus herpes gestacional e neonatal. Mostra Científica da Farmácia da Unicatólica. 2017; 4(1):1-2.

ALVES MMS, RODRIGUES BM, SANTOS JEB. A educação em saúde presente nos livros didáticos de ciências: uma abordagem sobre a promoção da saúde nos anos finais do ensino fundamental. Anais do 11. Encontro Internacional de Formação de Professores, 12. Fórum Permanente Internacional de Inovação Educacional, UNIT - Universidade Tiradentes, 2018.

AMABIS J, MARTHO GR. Biologia Moderna. v.1. São Paulo: Moderna, 2016.

BARDIN L. Análise de conteúdo. São Paulo: Edições, 2016.

BATISTA MVA, CUNHA MMS, CÂNDIDO AL. Análise do tema Virologia em livros didáticos de Biologia do ensino médio. Ensaio: Pesquisa em Educação em Ciências. 2010;12: 145-158.

BRASIL. Biblioteca Virtual em Saúde. Doenças sexualmente transmissíveis (DST). Publicado: 09 set. 2015. Disponível em: http://bvsms.saude.gov.br/dicas-em-saude/2063-doencassexualmente-transmissiveis-dst. Acesso em: 27 out. 2018

BRASIL. Ministério da Educação. Definição de critérios para avaliação dos livros didáticos: português, matemática, estudos sociais e ciências - $1^{\text {a }}$ a $4^{\text {a }}$ séries. Brasília: MEC, 1994. 378p.

BRASIL. Ministério da Educação. PNLD 2018: apresentação - guia de livros didáticos - ensino médio. Ministério da Educação - Secretária de Educação Básica - SEB - Fundo Nacional de Desenvolvimento da Educação. Brasília, DF: Ministério da Educação, Secretária de Educação Básica, 2017; 39p.

BRASIL. Ministério da Educação. PNLD 2018: apresentação - guia de livros didáticos - ensino médio. Ministério da Educação - Secretária de Educação Básica - SEB - Fundo Nacional de Desenvolvimento da Educação. Brasília, DF: Ministério da Educação, Secretária de Educação Básica, 2017. 39p.

BRASIL. Ministério da Educação. Secretaria de Educação Básica. Guia de livros didáticos: PNLD 2011. Brasília: MEC, 2010; 100p.

FREIRE P. Educação como prática da liberdade. Rio de Janeiro: Paz e Terra, 1967. 
BRASIL. Ministério da Educação. Secretaria de Educação Média e Tecnológica. PCNs + Ensino Médio: orientações educacionais complementares aos Parâmetros Curriculares Nacionais Ciências da Natureza, Matemática e suas Tecnologias. Brasília: MEC, 2002. 144p.

BRASIL. Ministério da Saúde. Departamento de DST, Aids e hepatites virais. O que você precisa saber sobre Aids. Ministério da Saúde: 2016. Disponível em: http://www.aids.gov.br/ptbr/publico-geral/o-que-e-hiv. Acesso em: 27 out. 2018.

BRASIL. Ministério da Saúde. Secretaria de Políticas em Saúde. Coordenação Nacional de DST e AIDS. Política Nacional de DST/aids: princípios e diretrizes. Brasília: Ministério da Saúde, 1999. Disponível em: http://bvsms.saude.gov.br/bvs/publicacoes/cd03_17.pdf. Acesso em: 07 jan. 2019.

BRASIL. Ministério da Saúde. Secretaria de Vigilância em Saúde. Guia prático sobre o HPV. Brasília, 2014. Disponível em:

http://portalarquivos.saude.gov.br/campanhas/2014/hpv/Guia_perguntas_e_repostas_MS_HPV_ profissionais_de_saude.pdf. Acesso em: 07 jan. 2019.

BRASIL. Ministério da Saúde. Vigilância, Prevenção e Controle das IST, do HIV/Aids e das Hepatites Virais. O que são IST. Disponível em: http://www.aids.gov.br/pt-br/publico-geral/oque-sao-ist. Acesso em: 27 out. 2018.

BRASIL. Secretaria de Educação Fundamental. Parâmetros Curriculares Nacionais: introdução aos parâmetros curriculares nacionais. Secretaria de Educação Fundamental. Brasília: MEC/SEF, 1997.126p.

BRASIL. Secretaria de Educação Fundamental. Parâmetros curriculares nacionais: terceiro e quarto ciclos: apresentação dos temas transversais. Secretaria de Educação Fundamental. Brasília: MEC/SEF, 1998. 436p.

CAMARGO FP, SILVA AFG, SANTOS ACA. A microbiologia no caderno do aluno e em livros didáticos: análise documental. Revista Ibero-americana de Educação, v.78, n.2, p.41-58, 2018.

CARNEIRO MHS, SANTOS WLP, MÓL GS. Livro didático inovador e professores: uma tensão a ser vencida. Ensaio Pesquisa em Educação em Ciências, v.7, n.2, p.1-14, 2005.

CASEMIRO JP, FONSECA, ABC, SECCO FVM. Promover saúde na escola: reflexões a partir de uma revisão sobre saúde escolar na América Latina. Ciência \& Saúde Coletiva. 2014; 19 (3):829-840.

CATANI A, CARVALHO EG, SANTOS FS, AGUILAR JB, CAMPOS SHA. Ser protagonista - biologia. v.1. São Paulo: SM, 2016.

COMPARINI R, DA SILVA ET, PEREIRA DCR. Estratégias de ampliação do diagnóstico da infecção pelo Vírus da Imunodeficiência Humana no Brasil, 2015.Comunicação em Ciências da Saúde. 2017; 28 (2):158-167. 
DE CICCO RR, VARGAS EP. As Doenças Sexualmente Transmissíveis em livros didáticos de biologia: aportes para o ensino de ciências. Revista Electrónica de Investigación en Educación en Ciencias. 2012; 7(1):10-21.

ESPINOLA CRR. Aves na escola: análise de livros didáticos do Ensino Fundamental. Trabalho de Conclusão de Curso, Universidade Federal de Santa Catarina, Florianópolis, SC, 2007.

FAVARETTO JA. Biologia unidade e diversidade. v.2. São Paulo: FTD, 2016.

LINHARES S, GEWANDSNAIDER F, PACCA H. Biologia hoje. v.1. São Paulo: Ática, 2016.

LOPES S, ROSSO S. Bio. v.3. São Paulo: Saraiva, 2016.

LUDOVICO RO, MAISTRO VIA. Sexualidade humana: um desafio nos livros didáticos. Enseñanza de las Ciencias, n. Extra. 2017: 5579-5583.

MARINHO JCB, SILVA JA, FERREIRA M. A educação em saúde como proposta transversal: analisando os Parâmetros Curriculares Nacionais e algumas concepções docentes. História, Ciências, Saúde - Manguinhos, Rio de Janeiro. 2015; 22 (2): 429-443.

MARTINS L, SANTOS GS, EL-HANI, CN. Abordagens de saúde em um livro didático de biologia largamente utilizado no ensino médio brasileiro. Investigações em Ensino de Ciências. 2012; $17(1): 249-283$.

MENDONÇA VL. Biologia. v.2 e 3. São Paulo: AJS, 2016.

MINAYO MCS, DESLANDES SF, CRUZ NETO O, GOMES R. Pesquisa social: teoria, método e criatividade. 21. ed. Petrópolis: Vozes, 2002.

MONTEIRO PHN, BIZZO N, GOUW AMS. As Doenças Sexualmente Transmissíveis (DST) e a Aids nos livros didáticos para o ensino fundamental no Brasil: abordagens e implicações educacionais. Acta Scientiae Canoas. 2010; 12 (1): 123-138.

MORAES R. Análise de conteúdo. Revista Educação, Porto Alegre, v.22, n.37, p.7-32, 1999.

MUNAKATA K. Livro didático como indício da cultura escolar. História da Educação, Santa Maria. 2016; 20 (50):119-138.

NEVES RC, RAMOS SIV. Educação Sexual nas Escolas: Educar para prevenir-estudo de caso. Arquivos de Psicologia: Portal dos Psicólogos. 2014: 1646-6977.

NICOLA JÁ, PANIZ CM. A importância da utilização de diferentes recursos didáticos no ensino de biologia. Infor: Inovação e Formação, São Paulo. 2016; 2 (1): 355-381.

OGO M, GODOY L. Contato biologia. v.1. São Paulo: Quinteto, 2016. 
OPAS/OMS. Organização Pan-Americana da Saúde. Organização Mundial da Saúde. HPV e câncer do colo do útero. Brasília, 2016, Disponível em:

https://www.paho.org/bra/index.php?option=com_content\&view=article\&id=5634:folha-

informativa-hpv-e-cancer-do-colo-do-utero\&ltemid=839. Acesso em: 08 jan. 2019.

PAES CCDC; PAIXÃO ANP. A importância da abordagem da educação em saúde: revisão de literatura. Revista De Educação Da Universidade Federal Do Vale Do São Francisco. 2016, 6(11). Disponível em: https://www.periodicos.univasf.edu.br/index.php/revasf/article/view/38 Acesso em: 02 dez.2020.

PALMA YA, PIASON SA, MANSO AG, STRE MN. Parâmetros curriculares nacionais: um estudo sobre orientação sexual, gênero e escola no Brasil. Temas em Psicologia, Ribeirão Preto. 2015; 23 (3): 727-738.

PEREIRA RMS, VALERIO FM, BARROS KM, SILVA REIS T, TRAJANO LT, SILVA LR. Conhecimento de acadêmicos da área de saúde sobre sífilis. Revista Práxis. 2018;10 (20):119-127.

ROCHA D, DEUSDARÁ B. Análise de conteúdo e análise do discurso: aproximações e afastamentos na (re)construção de uma trajetória. Alea, Rio de Janeiro. 2005; 7 (2): 305-322.

ROSA JL. Psicologia e educação o significado do aprender. 7.ed. Porto Alegre: EDIPUCRS, 2003.

ROSA MDA, MOHR A. Os fungos na escola: análise dos conteúdos de micologia em livros didáticos do ensino fundamental de Florianópolis. Experiências em Ensino de Ciências, Porto Alegre. 2010; 5 (3): 95-102.

SANTOS SCS, TERÁN AF, SILVA-FORSBERG MC. Analogias em livros didáticos de biologia no ensino de zoologia. Investigações em Ensino de Ciências. 2011; 15 (3): 591-603.

SÁ-SILVA JR, ALMEIDA CD, GUINDANI JF. Pesquisa documental: pistas teóricas e metodológicas. Revista Brasileira de História \& Ciências Sociais, ano I, n. I, p.1-15, 2009.

SCHIRMER SB, SAUERWEIN IPS. Livros didáticos em publicações na área de ensino: contribuições para análise e escolha. Investigações em ensino de ciências. 2017; 22 (1): 23-41.

SOUZA SL, COAN CM. Abordagem da sexualidade humana em livros didáticos de biologia. In: Anais do III Simpósio Internacional de Educação Sexual, Maringá. 2013: 24-26.

THOMPSON M, RIOS EP. Conexões com a Biologia. v.1. São Paulo: Moderna, 2016.

VASCONCELOS SD, SOUTO E. O livro didático de ciências no ensino fundamental proposta de critérios para análise do conteúdo zoológico. Ciência \& Educação. 2003; 9 (1): 93-104.

VILLEGAS-CASTANO A, TAMAYO-ACEVEDO LS. Prevalencia de infecciones de transmisión sexual y factores de riesgo para la salud sexual de adolescentes escolarizados, Medellín, Colombia, 2013. Iatreia, Medellín. 2016; 29 (1): 5-17. 


\section{CONTRIBUIÇÕES DE AUTORIA}

\section{1 - Halana Rafaela Alves da Silva}

Universidade Federal de Pernambuco, Graduada em Ciências Biológicas. https://orcid.org/0000-0002-1626-3580 - hallanaallves@gmail.com Contribuições: Elaborou o projeto, executou a metodologia da pesquisa, analisou os dados e redigiu o artigo.

\section{2 - Thaís Soares da Silva}

Universidade Federal Rural de Pernambuco, Doutoranda em Ensino de Ciências e Matemática pelo Programa de Pós Graduação em Ensino de Ciências e Matemática e Mestre em Educação pelo Programa de Pós Graduação em Educação.

https://orcid.org/0000-0003-2305-4645 - thais.soares1994@gmail.com

Contribuições: Auxiliou no desenvolvimento da pesquisa e contribuiu na avaliação crítica.

\section{3 - Gemilton de Freitas Mesquita}

Universidade Federal de Pernambuco, Mestre em Ensino de Biologia pelo Mestrado Profissional de Ensino em Biologia.

https://orcid.org/0000-0003-3278-7250 - gemiltonmesquita@gmail.com

Contribuições: Auxiliou no desenvolvimento da pesquisa e na análise de dados.

\section{4 - Isabella Macário Ferro Cavalcanti}

Universidade Federal de Pernambuco, Laboratório de Imunopatologia Keizo Asami. Laboratório de Microbiologia e Imunologia, Centro Acadêmico de Vitória, Professora Associada I das Disciplinas de Microbiologia, Imunologia e Exames Laboratoriais do Núcleo de Enfermagem.

https://orcid.org/0000000278893502 - isabella.cavalcanti@ufpe.br

Contribuições: Supervisionou o desenvolvimento da pesquisa e contribuiu na avaliação crítica.

\section{COMO CITAR ESTE ARTIGO}

SILVA, H. R. A.; SILVA, T. S.; MESQUITA, G. F.; CAVALCANTI, I. M. F. As Infecções Sexualmente Transmissíveis em livros didáticos de biologia do ensino médio: uma análise de conteúdo. Ciência e Natura, Santa Maria, v. 43, e43, p. 1-24, 2021. Disponível em: https://doi.org/10.5902/2179460X43923. Acesso em: dia, mês (abreviado), ano. 